\title{
COMMUTATORS MODULO THE CENTER IN A PROPERLY INFINITE VON NEUMANN ALGEBRA $\left({ }^{1}\right)$
}

\author{
BY \\ HERBERT HALPERN
}

1. Introduction. An element $C$ in a von Neumann algebra $\mathscr{A}$ is said to be a commutator in $\mathscr{A}$ if there are elements $A$ and $B$ in $\mathscr{A}$ such that $C=A B-B A$. For finite homogeneous discrete algebras and for properly infinite factor algebras the set of commutators has been completely described [1]-[5], [10]. In each of these special cases any element $C$ is a commutator modulo a central element depending on $C$. In this paper we show that given any element $C$ in a properly infinite von Neumann algebra $\mathscr{A}$ there is an element $C_{0}$ in the center of $\mathscr{A}$ depending on $C$ such that $C-C_{0}$ is a commutator in $\mathscr{A}$. The element $C_{0}$ is an arbitrary element in the intersection $\mathscr{K}_{C}$ of the center with the uniform closure of the convex hull of $\left\{U^{*} C U \mid U\right.$ unitary in $\left.\mathscr{A}\right\}$ [6, III, §5]. We then present a few facts about those elements $C$ such that $0 \in \mathscr{K}_{C}$ or what is the same as far as determining commutators is concerned about those elements $C$ such that $0 \in \mathscr{K}_{S^{-1} C S}$ for some invertible $S$ in $\mathscr{A}$.

2. Commutators. Let $\mathscr{A}$ be a $C^{*}$-algebra with identity and let $I$ be a closed two-sided ideal in $\mathscr{A}$. The image of the element $A \in \mathscr{A}$ in the factor algebra $\mathscr{A}(I)$ $=\mathscr{A} / I$ under the canonical homomorphism of $\mathscr{A}$ onto $\mathscr{A} / I$ will be denoted by $A(I)$. If $\zeta$ is a maximal ideal of the center of $\mathscr{A}$, the smallest closed two-sided ideal

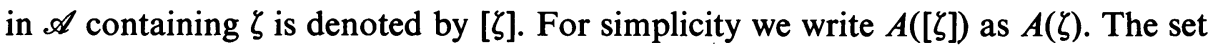
of maximal (respectively, primitive) ideals of $\mathscr{A}$ with the hull-kernel topology is called the strong structure space (respectively, structure space) of $\mathscr{A}$. If $\mathscr{A}$ is a von Neumann algebra, then the strong structure space $M(\mathscr{A})$ of $\mathscr{A}$ is homeomorphic with the spectrum of the center $\mathscr{Z}$ of $\mathscr{A}$ under the map $M \rightarrow M \cap \mathscr{Z}$ [13]. This means $M(\mathscr{A})$ is extremely disconnected.

Proposition 1. Let $\mathscr{A}$ be a properly infinite von Neumann algebra and let $A$ be a fixed element of $\mathscr{A}$. The function $M \rightarrow\|A(M)\|$ of the strong structure space $M(\mathscr{A})$ of $\mathscr{A}$ into the real numbers is continuous.

Proof. For every $\alpha \geqq 0$ we know that the set $X=\{M \in M(\mathscr{A}) \mid\|A(M)\| \leqq \alpha\}$ is closed. If $I=\bigcap X$, then $\|A(I)\| \leqq \alpha[8$, Lemma 1.9] and so $\|A(M)\| \leqq \alpha$ for every $M \in M(\mathscr{A})$ containing $I$. Thus $X=\{M \in M(\mathscr{A}) \mid I \subset M\}$.

Received by the editors September 27, 1968.

(1) The author was supported by the National Science Foundation.

Copyright (C) 1970, American Mathematical Society 
Conversely, let $\alpha>0$; we show that

$$
Y=\{M \in M(\mathscr{A}) \mid\|A(M)\| \geqq \alpha\}
$$

is closed in $M(\mathscr{A})$. Let $J$ be the strong radical of $\mathscr{A}$ and let $\mathscr{P}$ be the structure space of $\mathscr{A}(J)$. The set

$$
Y^{\prime}=\{K \in \mathscr{P} \mid\|A(J)(K)\| \geqq \alpha\}
$$

is compact (but not necessarily closed) in $\mathscr{P}[16,4.9 .18]$. If $\mathscr{P}^{\prime}$ is the structure space of $\mathscr{A}$, then $M \rightarrow M(J)$ defines a homeomorphism of $\left\{M \in \mathscr{P}^{\prime} \mid M \supset J\right\}=h(J)$ onto $\mathscr{P}[16,2.6 .6]$. But if $M \in \mathscr{P}^{\prime}$, then the intersection of $M$ with the center of $\mathscr{A}$ is a maximal ideal. So $M \in h(J)$ implies $M$ is of the form $J+[\zeta]$ for some maximal ideal $\zeta$ of the center. It is then clear that $h(J)$ is the set of maximal ideals of $\mathscr{A}$ [10, Proposition 2.3]. Furthermore, the topology of $h(J)$ and $M(\mathscr{A})$ coincide. This proves that $Y$ is compact in $M(\mathscr{A})$ since it is the inverse image of $Y^{\prime}$ under the homeomorphism $M \rightarrow M(J)$ of $M(\mathscr{A})$ onto $\mathscr{P}$. Because $M(\mathscr{A})$ is homeomorphic to the spectrum of the center which is Hausdorff, every compact set of $M(\mathscr{A})$ is closed. Thus $Y$ is a closed subset of $M(\mathscr{A})$. Q.E.D.

RemarK. If $\mathscr{A}$ is not properly infinite, Proposition 1 is certainly not true.

Let $H$ be a Hilbert space and let $A$ be a bounded linear operator on $H$. Let $F$ be a projection on $H$. Define the numerical gauge $\eta_{A}(F)$ to be

$$
\eta_{A}(F)=\operatorname{lub}\{\|A x-(A x, x) x\| \mid x \text { is a unit vector in } F(H)\} .
$$

Let $\mathscr{W}_{A}(F)$ be the closure of the convex set

$$
\{(A x, x) \mid x \text { a unit vector in } F(H)\} \text {. }
$$

For every $\alpha \in \mathscr{W}_{A}(F)$ we have that

$$
\|(A-\alpha) F\| \leqq 65 \eta_{A}(F) .
$$

This can be obtained by a simple reworking of Lemma 2.3 [2].

Let $\mathscr{A}$ be a properly infinite von Neumann algebra with no $\sigma$-finite type III direct summands; then for each projection $F$ in $\mathscr{A}$ and each element $A$ in $\mathscr{A}$ define $\nu_{A}(F)$ to be

$$
\nu_{A}(F)=\operatorname{lub}\{\|A E-E A E\| E \in(J), E \leqq F\}
$$

where $(J)$ is the set of projections in the strong radical $J$ of $\mathscr{A}$. For every irreducible representation $\phi$ of $\mathscr{A}$ on a Hilbert space such that $\phi(J) \neq(0)$ we have that

$$
\eta_{\phi(A)}(\phi(F)) \leqq \nu_{A}(F)
$$

[10, Proposition 3.1]. Define $\nu(A)$ to be

$$
\nu(A)=\operatorname{glb}\left\{\nu_{A}(F) \mid 1-F \in(J)\right\} .
$$


Let $\mathscr{A}$ now be the product of $\sigma$-finite type III algebras; let

$$
\nu(A)=\operatorname{lub}\{\|A E-E A E\| \mid E \text { a projection in } \mathscr{A}\}
$$

for each $A \in \mathscr{A}$. If $A$ is in the complement in $\mathscr{A}$ of the set of all elements of $\mathscr{A}$ equal to scalar (zero included) multiples of the identity modulo some maximal ideal of $\mathscr{A}$, then there is a $\nu>0$ such that $\nu(A P) \geqq \nu$ for every nonzero central projection $P$ since there is a projection $E$ in $\mathscr{A}$ with $E \sim 1-E \sim 1$ such that $E A^{*}(1-E) A E$ $\geqq \alpha E$ for some scalar $\alpha>0$ [10, Theorem 3.7]. Also it is easy to see from Proposition $3.1[10]$ that

$$
\eta_{\phi(A)}(1) \leqq \nu(A)
$$

for every irreducible representation of $\mathscr{A}$.

LEMMA 2. Let $\mathscr{A}$ be a properly infinite von Neumann algebra such that

(i) either $\mathscr{A}$ has no $\sigma$-finite type III direct summands or

(ii) $\mathscr{A}$ is a product of $\sigma$-finite type III algebras.

Let $\left\{P_{n}\right\}$ be a set of mutually orthogonal central projections of $\mathscr{A}$ of sum $P$. Then $\nu(A P)=\operatorname{lub}_{n} \nu\left(A P_{n}\right)$ for every $A \in \mathscr{A}$.

Proof. Suppose $\mathscr{A}$ satisfies condition (i). Let $(J)$ be the set of projections in the strong radical of $\mathscr{A}$. Then given $\varepsilon>0$ there is a projection $F$ with $1-F \in(J)$ such that

But

$$
\nu(A P)+\varepsilon \geqq \operatorname{lub}\{\|(1-E) A P E\| \mid E \leqq F, E \in(J)\} .
$$

$$
\left\|(1-E) A E P_{n}\right\| \leqq\|(1-E) A P E\|
$$

for every $P_{n}$. So $\nu\left(A P_{n}\right) \leqq \nu(A P)+\varepsilon$ for every $n$. Since $\varepsilon>0$ is arbitrary, we have that lub $\nu\left(A P_{n}\right) \leqq \nu(A P)$. Conversely, given that $\varepsilon>0$ there is for each $P_{n}$ a projection $F_{n}$ with $1-F_{n} \in(J)$ such that

$$
\operatorname{lub}\left\{\left\|(1-E) A P_{n} E\right\| \mid E \leqq F_{n}, E \in(J)\right\} \leqq \nu\left(A P_{n}\right)+\varepsilon .
$$

Setting $F=\sum F_{n} P_{n}$, we have that $P-F \in(J)$ [10, Corollary, Proposition 2.2] and that

$$
\|(1-E) A P E\|=\operatorname{lub}_{n}\left\|(1-E) A P_{n} E\right\| \leqq \operatorname{lub} \nu\left(A P_{n}\right)+\varepsilon
$$

for every $E$ in $(J)$ with $E \leqq F$. Thus $\nu(A P) \leqq \operatorname{lub} \nu\left(A P_{n}\right)+\varepsilon$. Since $\varepsilon>0$ is arbitrary, we have that $\nu(A P) \leqq$ lub $\nu\left(A P_{n}\right)$. This completes the proof for case (i).

The proof for case (ii) is similar.

LEMMA 3. Let $\mathscr{A}$ be a properly infinite von Neumann algebra with no $\sigma$-finite type III direct summands. Let $G$ be a projection in $\mathscr{A}$ such that the orthogonal complement $1-G$ of $G$ is in the set of projections $(J)$ of the strong radical of $\mathscr{A}$. Let $\left(J^{\prime}\right)$ be the strong radical of the reduced algebra GAAG. For any element $A$ in $\mathscr{A}$ we have that

$$
\nu^{\prime}(G A G)=\operatorname{glb}\left\{\operatorname{lub}\left\{\|(1-E) G A G E\| \mid E \leqq F, E \in\left(J^{\prime}\right)\right\} \mid G-F \in\left(J^{\prime}\right)\right\}
$$

is equal to $\nu(A)$. 
Proof. First we show that $\left(J^{\prime}\right)=\{E \in(J) \mid E \leqq G\}$. Suppose $E \notin\left(J^{\prime}\right)$ for some $E \in(J)$ majorized by $G$. There is a nonzero projection $P$ in the center of $G \mathscr{A} G$ such that $P E \sim P[10, \S 2]$. But $P=Q G$ for some nonzero $Q$ in the center of $\mathscr{A}$ [6, I, §2, Corollary, Proposition 2]. Then $Q E \sim Q G \sim Q$ since $G \sim 1$ [cf. 10, §2]. So $E \notin(J)$. This is a contradiction. Hence $\{E \in(J) \mid E \leqq G\} \subset\left(J^{\prime}\right)$. Conversely, suppose $E \in\left(J^{\prime}\right)$. If $Q$ is a central projection in $\mathscr{A}$ with $E Q \sim Q$, then $E Q \sim Q \sim Q G$. This implies $Q=0$. So $\left(J^{\prime}\right) \subset\{E \in(J) \mid E \leqq G\}$.

Now let $\varepsilon>0$ be given. There is by relation (2) a projection $F$ in $\mathscr{A}$ with $1-F \in(J)$ such that $\nu_{A}(F) \leqq \nu(A)+\varepsilon$. Let $G^{\prime}$ be the least upper bound of $1-G$ and $1-F$. Then $G^{\prime} \in(J)$ and $1-G^{\prime} \leqq F$ and $1-G^{\prime} \leqq G$. We see that

$$
G-\left(1-G^{\prime}\right)=G^{\prime}-(1-G) \in\left(J^{\prime}\right)
$$

by the first paragraph. So

$$
\begin{aligned}
\nu^{\prime}(G A G) & \leqq \operatorname{lub}\left\{\|(1-E) G A G E\| \mid E \in\left(J^{\prime}\right), E \leqq 1-G^{\prime}\right\} \\
& =\operatorname{lub}\left\{\|G(1-E) A E\| \mid E \in(J), E \leqq 1-G^{\prime}\right\} \\
& \leqq \operatorname{lub}\{\|(1-E) A E\| \mid E \in(J), E \leqq F\} \leqq \nu(A)+\varepsilon .
\end{aligned}
$$

Since $\varepsilon>0$ is arbitrary, we have that $\nu^{\prime}(G A G) \leqq \nu(A)$.

Conversely, let $\varepsilon>0$ be given; there is a projection $F \in G \mathscr{A} G$ with $G-F \in\left(J^{\prime}\right)$ and

$$
\operatorname{lub}\left\{\|(G-E) A E\| \mid E \leqq F, E \in\left(J^{\prime}\right)\right\} \leqq \nu^{\prime}(G A G)+\varepsilon .
$$

The domain support $G^{\prime}$ of $(1-G) A$ is a projection in $(J)$ since $G^{\prime} \prec 1-G$ [6, III, $\S 1$, Proposition 2]; similarly, the domain support $G^{\prime \prime}$ of $G^{\prime} F$ is a projection in $(J)$ majorized by $F$. So $1-\left(F-G^{\prime \prime}\right)=(1-G)+(G-F)+G^{\prime \prime}$ is a projection in $(J)$. Then

But

$$
\nu(A) \leqq \operatorname{lub}\left\{\|(1-E) A E\| \mid E \leqq F-G^{\prime \prime}, E \in(J)\right\} .
$$

$G A G E=A E-(1-G) A E=A E-(1-G) A G^{\prime} F E=A E-(1-G) A G^{\prime} F G^{\prime \prime} E=A E$.

So we see that

$$
\begin{aligned}
\nu(A) & \leqq \operatorname{lub}\left\{\|(1-E) G A G E\| \mid E \leqq F-G^{\prime \prime}, E \in\left(J^{\prime}\right)\right\} \\
& \leqq \operatorname{lub}\left\{\|(1-E) G A G E\| \mid E \leqq F, E \in\left(J^{\prime}\right)\right\} \leqq \nu^{\prime}(G A G)+\varepsilon .
\end{aligned}
$$

Since $\varepsilon>0$ is arbitrary, we have that $\nu(A) \leqq \nu^{\prime}(G A G)$. Q.E.D.

THEOREM 4. Let $C$ be an element in a properly infinite von Neumann algebra $\mathscr{A}$ and let $C_{0}$ be an element in the intersection $\mathscr{K}_{C}$ of the center of $\mathscr{A}$ with the uniform closure of the convex hull of $\left\{U^{*} C U \mid U\right.$ unitary in $\left.\mathscr{A}\right\}$. Then $C-C_{0}$ is a commutator in $\mathscr{A}$.

Proof. We first make a preliminary reduction. There is a maximal central projection $P$ in $\mathscr{A}$ such that $\left(C-C_{0}\right) P$ is in the strong radical $J$ of $\mathscr{A}[10$, Corollary, 
Proposition 2.2]. However, the structure of the strong radical of $\mathscr{A}$ allows us to conclude that $\left(C-C_{0}\right) P$ is in the strong radical of $\mathscr{A} P[10, \S 2]$. Since $\left(C-C_{0}\right) P$ is a commutator in $\mathscr{A} P$, it is necessary to prove that $\left(C-C_{0}\right)(1-P)$ is a commutator in $\mathscr{A}(1-P)$. It is easy to see that the uniform closure of the convex hull of $\left\{U^{*}\left(C-C_{0}\right) U \mid U\right.$ unitary in $\left.\mathscr{A}(1-P)\right\}$ contains 0 . So without loss of generality we may assume that $C$ is an element in $\mathscr{A}$ such that $0 \in \mathscr{K}_{C}$ and such that $C P \in J$ for some central projection $P$ in $\mathscr{A}$ implies that $P=0$.

Now there is a central projection $Q$ in $\mathscr{A}$ such that

(i) $\mathscr{A} Q$ has no $\sigma$-finite type III direct summands and

(ii) $\mathscr{A}(1-Q)$ is the product of $\sigma$-finite type III algebras.

It is clearly necessary to prove only that $C Q$ and $C(1-Q)$ are commutators in $\mathscr{A} Q$ and $\mathscr{A}(1-Q)$ respectively. Here $0 \in \mathscr{K}_{C Q}$ and $0 \in \mathscr{K}_{C(1-Q)}$ is also true when these sets are formed relative to $\mathscr{A} Q$ and $\mathscr{A}(1-Q)$ respectively. In the ensuing paragraphs we shall assume that either $\mathscr{A}$ satisfies condition (i) or condition (ii).

Let $\|C\|=\alpha$. We construct by induction a sequence $\left\{P_{n}\right\}$ of mutually orthogonal central projections of sum 1 such that for each nonzero central projection $P$ majorized by $P_{n}$ the number $\nu(C P)$ lies in the real interval $\left[2^{-n} \alpha, 2^{-n+1} \alpha\right]$. The induction hypothesis may be stated as follows: let $P_{0}=0$; then $P_{n}$ is the largest central projection majorized by $1-\sum\left\{P_{k} \mid 0 \leqq k \leqq n-1\right\}$ such that $\nu(C P) \in I_{n}$ for every nonzero central projection $P$ majorized by $P_{n}$. Suppose we have constructed $P_{1}, P_{2}, \ldots, P_{n}$. We find $P_{n+1}$. We may assume $R=1-\sum\left\{P_{k} \mid 0 \leqq k \leqq n\right\}$ is nonzero. There is a maximal ideal $M$ in the strong structure space $M(\mathscr{A})$ of $\mathscr{A}$ such that $C R(M)$ is not in the center of $\mathscr{A}(M)$. Indeed, suppose $C R(M)$ is in the center of $\mathscr{A}(M)$ for every $M \in M(\mathscr{A})$. Given $\varepsilon>0$ there are unitary operators $U_{1}, U_{2}, \ldots$, $U_{m}$ in $\mathscr{A}$ and positive scalars $\alpha_{1}, \alpha_{2}, \ldots, \alpha_{m}$ of sum 1 such that $\left\|\sum \alpha_{i} U_{i}^{*} C U_{i}\right\|<\varepsilon$ since $0 \in \mathscr{K}_{C}$. Thus

$$
\|C R(M)\|=\left\|\left(\sum \alpha_{i} U_{i}^{*} C U_{i}\right)(M)\right\|<\varepsilon
$$

for every $M \in M(\mathscr{A})$. Because $\varepsilon>0$ is arbitrary, we have that $C R(M)=0$ for every $M \in M(\mathscr{A})$. This means that $C R$ is in the strong radical of $\mathscr{A}$. This is contrary to the choice of $C$. Hence, we must conclude that $C R(M)$ is not in the center of $\mathscr{A}(M)$ for at least one $M$ in $M(\mathscr{A})$. Then there is a projection $E$ in $\mathscr{A}$ such that $\|(1-E) C R E(M)\| \neq 0$. By the continuity of $M^{\prime} \rightarrow\left\|(1-E) C R E\left(M^{\prime}\right)\right\|$ on $M(\mathscr{A})$ (Proposition 1), there is an open and closed neighborhood $X$ of $M$ in $M(\mathscr{A})$ such that for every $M^{\prime} \in X$ the element $C R\left(M^{\prime}\right)$ is not in the center of $\mathscr{A}\left(M^{\prime}\right)$. Let $Q$ be the nonzero central projection of $\mathscr{A}$ which determines $X$ by the relation $X=\left\{M^{\prime} \in M(\mathscr{A}) \mid Q \notin M^{\prime}\right\}$ [13]. Then $Q$ is majorized by $R$ and $C Q\left(M^{\prime}\right)$ is not in the center of $\mathscr{A} Q\left(M^{\prime}\right)$ for every $M^{\prime}$ in the strong structure space $M(\mathscr{A} Q)$ of the algebra $\mathscr{A} Q$. The latter is true because $M^{\prime} \rightarrow M^{\prime} Q$ defines a homeomorphism of

$$
\left\{M^{\prime} \in M(\mathscr{A}) \mid Q \notin M^{\prime}\right\} \text { onto } M(\mathscr{A} Q) \text { [16, Theorem 2.6.6]. }
$$


Then by 3.1 and 3.7 [10] there is a $\nu>0$ such that $\nu\left(C Q^{\prime}\right) \geqq \nu$ for every nonzero central projection $Q^{\prime}$ majorized by $Q$. It is clearly immaterial whether $v\left(C Q^{\prime}\right)$ is evaluated in $\mathscr{A} Q$ or in $\mathscr{A}$. Let $m$ be the smallest integer for which there is a nonzero central projection $Q$ majorized by $R$ such that $\nu\left(C Q^{\prime}\right) \geqq 2^{-m} \alpha$ for every nonzero central projection $Q^{\prime}$ majorized by $Q$. We then have that $\nu\left(C Q^{\prime}\right) \in I_{m}$ for every such $Q^{\prime}$. In fact by the choice of $m$ the projection $Q$ is easily seen to be the least upper bound of a set $\left\{Q_{i}\right\}$ of nonzero mutually orthogonal central projections which satisfy $2^{-m} \alpha \leqq \nu\left(C Q_{i}\right)<2^{-m+1} \alpha$. By Lemma 2 we may conclude that $\nu(C Q) \leqq$ $2^{-m+1} \alpha$. So for every nonzero central projection $Q^{\prime}$ majorized by $Q$ we have that $\nu\left(C Q^{\prime}\right) \leqq \nu(C Q) \leqq 2^{-m+1} \alpha$. Now if $n+1<m$ set $P_{n+1}$ equal to zero. If $m \leqq n+1$, then $m=n+1$. Indeed suppose that $m<n+1$; the projection $P_{m}+Q \neq P_{m}$, and for any nonzero central projection $Q^{\prime}$ majorized by $P_{m}+Q$ we have that

$$
\nu\left(C Q^{\prime}\right)=\operatorname{lub}\left\{\nu\left(C P_{m} Q^{\prime}\right), \nu\left(C Q Q^{\prime}\right)\right\} \in I_{m} .
$$

This contradicts the definition of $P_{m}$. Therefore $m=n+1$. Now we argue as follows. Let $\left\{Q_{n}\right\}$ be a maximal set of nonzero mutually orthogonal central projections majorized by $R$ such that $\nu\left(C Q^{\prime}\right) \in I_{n+1}$ for every nonzero central projection $Q^{\prime}$ majorized by some $Q_{i}$. Let $P_{n+1}=\sum Q_{i}$. It is clear that $P_{n+1} \leqq R$. Because $\nu(C Q)$ $=\operatorname{lub}_{i} \nu\left(C Q Q_{i}\right)$ for any nonzero central projection $Q$ majorized by $P_{n+1}$ (Lemma 2) and since at least one projection $Q Q_{i}$ is nonzero, we have that $v(C Q) \geqq 2^{-(n+1)} \alpha$. On the other hand $Q \leqq R$ and so by the induction hypothesis there is a set $\left\{R_{i}\right\}$ of nonzero mutually orthogonal central projections of sum $Q$ such that $\nu\left(C R_{i}\right)<2^{-n} \alpha$ for each $R_{i}$. Thus $\nu(C Q) \leqq 2^{-n} \alpha$ (Lemma 2). This proves that $\nu(C Q) \in I_{n+1}$. It is clear that $P_{n+1}$ is the largest central projection majorized by $R$ such that $\nu(C Q)$ $\in I_{n+1}$ for every nonzero central projection majorized by $P_{n+1}$.

Suppose that the sequence $\left\{P_{n}\right\}$ with the required properties has been constructed by induction. We show that $\sum P_{n}=1$. If $R=1-\sum P_{n}$, then for each $n=1,2, \ldots$ we may conclude that $\nu(C R) \leqq 2^{-n} \alpha$ by performing the construction of the previous paragraph. This means that $\nu(C R)=0$. The results of the previous paragraph show that $R=0$ by our choice of $C$. Hence $\sum P_{n}=1$.

Let $Z$ be the spectrum of the center of $\mathscr{A}$ and let $X_{n}=\left\{\zeta \in Z \mid P_{n} \notin \zeta\right\}$ for $n=1,2, \ldots$ Now suppose that $\mathscr{A}$ has no $\sigma$-finite type III direct summands. For each $n=1,2, \ldots$ there is a projection $F_{n}$ in $\mathscr{A} P_{n}$ with $P_{n}-F_{n} \in J$ such that $\nu_{C P_{n}}\left(F_{n}\right)$ $\leqq 2 \nu\left(C P_{n}\right)$ by definition. For each $\zeta \in Z$ there is an irreducible representation $\psi_{\zeta}=\psi$ of $\mathscr{A}$ on a Hilbert space whose kernel is the smallest closed two-sided ideal

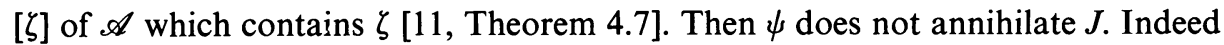
$J$ contains a projection $E$ of central support 1, cf. [10, §2]. The two-valued continuous function $\zeta^{\prime} \rightarrow\left\|E\left(\zeta^{\prime}\right)\right\|[9$, Lemma 10] on $Z$ assumes the value 0 on an open and closed set given by $\left\{\zeta^{\prime} \in Z \mid R \notin \zeta^{\prime}\right\}$ where $R$ is a central projection. Then $\left\|(E(1-R)-E)\left(\zeta^{\prime}\right)\right\|=0$ for every $\zeta^{\prime} \in Z$. Since $\bigcap\left\{\left[\zeta^{\prime}\right] \mid \zeta^{\prime} \in Z\right\}=(0)$ cf. [9, $\S 4$, remarks preceding Lemma 9], we have that $E(1-R)=E$. But this means that $R=0$ since the central support of $E$ is 1 . This proves that $E\left(\zeta^{\prime}\right) \neq 0$ for every $\zeta^{\prime} \in Z$ 
and in particular $E(\zeta) \neq 0$. So $\psi$ does not annihilate $J$. Thus relations (1) and (3) imply that

$$
\left\|\left(\psi\left(C P_{n}\right)-\beta\right) \psi\left(F_{n}\right)\right\| \leqq 65 \eta_{\psi\left(C P_{n}\right)}\left(\psi\left(F_{n}\right)\right) \leqq 65 v_{C P_{n}}\left(F_{n}\right) \leqq 130 \nu\left(C P_{n}\right)
$$

whenever $\beta \in \mathscr{W}_{\psi\left(C P_{n}\right)}\left(\psi\left(F_{n}\right)\right)=\mathscr{W}$ and $\zeta \in X_{n}$. We show that $0 \in \mathscr{W}$. Indeed, given $\varepsilon>0$, there is a set $U_{1}, U_{2}, \ldots, U_{m}$ of unitary elements in $\mathscr{A}$ and positive scalars $\alpha_{1}, \alpha_{2}, \ldots, \alpha_{m}$ of sum 1 such that $\left\|\sum \alpha_{i} U_{i}^{*} C U_{i}\right\|<\varepsilon$. If $G_{i}$ is the range projection of $U_{i}^{*}\left(P_{n}-F_{n}\right)$ for $i=1,2, \ldots, m$, then $G_{i}$ is a projection in $J$ and $G=\operatorname{lub} G_{i}$ is a projection in $J$. Thus the projection $P_{n}-G$ is equivalent to $P_{n}$. Let $x$ be a unit vector in the subspace determined by $\psi\left(P_{n}-G\right)$. For each $U_{i}$ we have that $\psi\left(U_{i}\right) x$ $=y_{i}$ is in the orthogonal complement of the subspace determined by $\psi\left(P_{n}-F_{n}\right)$ and thus $\psi\left(F_{n}\right) y_{i}=y_{i}$. Therefore

$$
\sum \alpha_{i}\left(\psi\left(C P_{n}\right) y_{i}, y_{i}\right) \in \mathscr{W}
$$

since $\mathscr{W}$ is convex. But we have that

$$
\left|\sum\left(\alpha_{i} \psi\left(C P_{n}\right) y_{i}, y_{i}\right)\right| \leqq\|\psi\|\left\|\sum \alpha_{i} U_{i}^{*} C U_{i}\right\|\|x\|^{2}<\varepsilon .
$$

Because $\mathscr{W}$ is closed and because $\varepsilon>0$ is arbitrary, we see that $0 \in W$. The relation (5) now becomes

$$
\left\|C P_{n} F_{n}(\zeta)\right\|=\left\|C F_{n}(\zeta)\right\|=\left\|\psi_{\zeta}\left(C P_{n}\right) \psi_{\zeta}\left(F_{n}\right)\right\| \leqq 130 \nu\left(C P_{n}\right),
$$

for every $\zeta \in X_{n}$. The orthogonal complement of the projection $F=\sum F_{n}$ is in $J$ since $P_{n}(1-F) \in J$ for every $n=1,2, \ldots$ [10, Proposition 2.2]. For every nonzero central projection $P$ majorized by $P_{n}$ we have that

$$
\|C F P\|=\operatorname{lub}\left\{\|(C F(\zeta) \| \mid P \notin \zeta, \zeta \in Z\} \leqq 130 v\left(C P_{n}\right) \leqq 260 \nu(C P) .\right.
$$

So for any central projection $P$ we have that

$$
\begin{aligned}
\|C F P\| & =\operatorname{lub}\left\{\left\|C F P P_{n}\right\| \mid n=1,2, \ldots\right\} \\
& \leqq \operatorname{lub}\left\{260 \nu\left(C P P_{n}\right) \mid n=1,2, \ldots\right\} \\
& \leqq 260 v(C P)
\end{aligned}
$$

by Lemma 2.

Now for an algebra which is the product of $\sigma$-finite type III algebras, we may show that $\|C P\| \leqq 260 \nu(C P)$ for any central projection $P$. The proof is entirely similar to that just given except that relation (4) replaces relations (1) and (3).

Now let us suppose that $\mathscr{A}$ has no $\sigma$-finite type III direct summands. Let $D=F C F$ and let $\mathscr{B}$ be the von Neumann algebra $F \mathscr{A} F$. By setting $Q_{n}=P_{n} F$ we obtain a sequence $\left\{Q_{n}\right\}$ of mutually orthogonal central projections in $\mathscr{B}$ of sum $F$ such that $\nu(D Q) \in I_{n}$ for any nonzero central projection $Q$ in $\mathscr{B}$ majorized by $Q_{n}$. Here $\nu(D Q)$ is evaluated in $\mathscr{B}$ and Lemma 3 is employed. By relation (6) we see that 
$\left\{\left\|2^{n} D Q_{n}\right\|\right\}$ is a bounded sequence and hence $B=\sum 2^{n} D Q_{n}$ defines an element of $\mathscr{B}$ such that $\nu(B Q) \geqq \alpha$ for every nonzero central projection $Q$ in $\mathscr{B}$. Indeed,

$$
\begin{aligned}
\nu(B Q) & =\operatorname{lub}\left\{\nu\left(B Q Q_{n}\right) \mid n=1,2, \ldots\right\} \\
& =\operatorname{lub}\left\{2^{n} \nu\left(D Q Q_{n}\right) \mid n=1,2, \ldots\right\} \geqq \alpha
\end{aligned}
$$

since at least one projection $Q Q_{n}$ is nonzero. There is an invertible element $S$ in $\mathscr{B}$ and a projection $G$ in $\mathscr{B}$ with $F \sim G \sim F-G$ such that $U^{*} S^{-1} B S U=0$ and $V^{*} S^{-1} B S V$ is a commutator in $\mathscr{B}$. Here $U$ and $V$ are partial isometries in $B$ such that $U^{*} U=V^{*} V=F, U U^{*}=G$ and $V V^{*}=F-G$ [10, Theorem 3.6]. By multiplying both $U^{*} S^{-1} B S U$ and $V^{*} S^{-1} B S V$ by the central element $\sum 2^{-n} Q_{n}$ we see that $U^{*} S^{-1} D S U=0$ and $V^{*} S^{-1} D S V$ is a commutator in $\mathscr{B}$. Now let $T=S+(1-F)$ in $\mathscr{A}$; the element $T$ is invertible with inverse $T^{-1}=S^{-1}+(1-F)$ where $S^{-1}$ still denotes the inverse of $S$ in $\mathscr{B}$. Let $W$ be a partial isometry in $\mathscr{A}$ with domain support 1 and range support $F$. Then $V_{1}=V W$ is a partial isometric operator of domain support 1 and range support $F-G$. Then it is easy to see that $V_{1}^{*} T^{-1} C T V_{1}$ $=W^{*} V^{*} S^{-1} D S V W$ is a commutator in $\mathscr{A}$. We have that $1 \sim F \sim G \prec G+(1-F)$. Thus there is a partial isometry $U_{1}$ in $\mathscr{A}$ with domain support 1 and range support $G+(1-F)$. Then

$$
U_{1}^{*} T^{-1} C T U_{1}=U_{1}^{*} T^{-1} C T(1-F) U_{1}+U_{1}^{*}(1-F) T^{-1} C T G U_{1}
$$

is an element of the strong radical of $\mathscr{A}$ and therefore, is a commutator in $\mathscr{A}[10$, Theorem 2.5]. We have proved that there is an isomorphism of $\mathscr{A}$ onto the algebra $\mathscr{A}_{2}$ of $2 \times 2$ matrices over $\mathscr{A}$ which carries $T^{-1} C T$ into the matrix $\left(B_{i j}\right)$ where $B_{11}$ and $B_{22}$ are commutators in $\mathscr{A}$. But this matrix is a commutator in the algebra $\mathscr{A}_{2}$. Indeed, let $B_{11}=S_{11} T_{11}-T_{11} S_{11}$ and $B_{22}=S_{22} T_{22}-T_{22} S_{22}$ for $S_{11}, S_{22}, T_{11}$, $T_{22}$ in $\mathscr{A}$. We may assume that $S_{11}$ and $S_{22}$ have disjoint spectra since $B_{11}=$ $\left(S_{11}+\beta\right) T_{11}-T_{11}\left(S_{11}+\beta\right)$ for a scalar $\beta$. There is an operator $T_{12}$ and an operator $T_{21}$ in $\mathscr{A}$ such that $S_{11} T_{12}-T_{12} S_{22}=B_{12}$ and $S_{22} T_{21}-T_{21} S_{11}=B_{21}$ [12]. Setting $S_{21}=S_{12}=0$, we find by direct calculation that $\left(S_{i j}\right)\left(T_{i j}\right)-\left(T_{i j}\right)\left(S_{i j}\right)=\left(B_{i j}\right)$ in $\mathscr{A}_{2}$. This proves $\left(B_{i j}\right)$ is a commutator in $\mathscr{A}_{2}$ and $T^{-1} C T$ is a commutator in $\mathscr{A}$.

Now if $\mathscr{A}$ is the product of $\sigma$-finite type III agebras, the preceding paragraph allows us to conclude that there is an invertible $S$ in $\mathscr{A}$ such that $S^{-1} C S$ may be identified with the $2 \times 2$ matrix $\left(B_{i j}\right)$ over $\mathscr{A}$ with $B_{11}=0$ and $B_{22}$ a commutator in $\mathscr{A}$. So $\left(B_{i j}\right)$ is a commutator in the $2 \times 2$ matrices over $\mathscr{A}$ and $S^{-1} C S$ is a commutator in $\mathscr{A}$. Q.E.D.

3. Elements $C$ with $0 \in \mathscr{K}_{C}$. The construction of Theorem 4 actually depended upon choosing a central element $C_{0}$ corresponding to a given element $C$ in a properly infinite von Neumann algebra $\mathscr{A}$ such that $E S^{-1}\left(C-C_{0}\right) S E=0$ for some invertible $S$ in $\mathscr{A}$ and some projection $E$ in $\mathscr{A}$ equivalent to 1 . The next proposition clarifies this choice. 
Proposition 5. If $C$ is an element in a properly infinite von Neumann algebra $\mathscr{A}$ such that $E C E=0$ for some projection $E$ in $\mathscr{A}$ equivalent to 1 , then 0 is an element of the intersection $\mathscr{K}_{C}$ of the center of $\mathscr{A}$ with the uniform closure of the convex hull of the set $\left\{U^{*} C U \mid U\right.$ unitary in $\left.\mathscr{A}\right\}$.

Proof. There are projections $E^{\prime}$ and $E^{\prime \prime}$ such that $E^{\prime} \sim E^{\prime \prime} \sim E$ and $E^{\prime}+E^{\prime \prime}=E$ [6, III, $\S 8$, Theorem 1, Corollary 2]. Then $1 \sim E^{\prime} \leqq E^{\prime}+(1-E) \leqq 1$ implies that $E^{\prime}+(1-E) \sim 1$. So there is no loss of generality in supposing that $E \sim 1-E \sim 1$. The operator $U=E-(1-E)$ is unitary in $\mathscr{A}$ and

$$
2^{-1}\left(C+U^{*} C U\right)=(1-E) C(1-E) \text {. }
$$

Now let $E_{1}, E_{2}, \ldots, E_{n}$ be orthogonal projections of sum $E$ such that $E_{1} \sim \ldots$ $\sim E_{n} \sim E$. There are unitary operators $U_{1}, U_{2}, \ldots, U_{n}$ in $\mathscr{A}$ such that $(1-E) U_{j}$ has domain support $E_{j}$ for $j=1,2, \ldots, n$ since $E_{j} \sim 1-E$ and $1-E_{j} \sim E$ for $j=1,2$, $\ldots, n$. So we have that

$$
\begin{array}{r}
\left\|\sum\left\{n^{-1} U_{j}^{*}(1-E) C(1-E) U_{j} x \mid j=1,2, \ldots, n\right\}\right\|^{2} \\
\leqq n^{-2}\|C\|^{2} \sum\left\|E_{j} x\right\|^{2} \leqq n^{-2}\|C\|^{2}\|x\|^{2}
\end{array}
$$

for every $x$ in the Hilbert space. Thus

$$
\left\|\sum n^{-1} U_{j}^{*}(1-E) C(1-E) U_{j}\right\| \leqq n^{-1}\|C\| .
$$

This means that 0 is an element of the uniform closure of the convex hull of $\left\{V^{*} C V \mid V\right.$ unitary in $\left.\mathscr{A}\right\}$ because $n$ is arbitrary. Hence $0 \in \mathscr{K}_{C}$. Q.E.D.

Let $\mathscr{A}$ be a properly infinite von Neumann algebra. If we could prove that $0 \in \bigcup\left\{\mathscr{K}_{S^{-1} C S} \mid S\right.$ invertible in $\left.\mathscr{A}\right\}$ for every commutator $C$ in $\mathscr{A}$, then we would have a complete characterization of the set commutators. This characterization is certainly valid for factor algebras. Indeed $C$ is a commutator in the properly infinite factor algebra $\mathscr{A}$ if and only if $\mathscr{A}$ is not a nonzero scalar multiple of the identity modulo the unique maximal ideal $M$ of $\mathscr{A}$. If $C \in M$, then $0 \in \mathscr{K}_{C}[10$, Proposition 2.4]. If $C$ is not a scalar multiple of the identity modulo $M$, then the canonical form of Brown and Pearcy [2] in conjunction with the preceding proposition shows $0 \in \mathscr{K}_{S^{-1} C S}$ for some invertible $S$ in $\mathscr{A}$. The characterization though is at odds with a conjecture that the set of commutators in $\mathscr{A}$ is the complement $\left(F^{\prime}\right)$ in $\mathscr{A}$ of the set of all elements equal to a nonzero scalar multiple of the identity modulo some maximal ideal of $\mathscr{A}$ [4]. In fact let $\left\{P_{n}\right\}$ be a sequence of nonzero mutually orthogonal central projections of sum 1. (This presupposes that $\mathscr{A}$ has a sufficiently large center.) Then let $E_{n}$ be a projection in $\mathscr{A} P_{n}$ such that $E_{n} \sim P_{n}$ $\sim P_{n}-E_{n}(n=1,2, \ldots)$. Let $C=\sum\left(n^{-1} P_{n}+n^{-2} E_{n}\right)$. We have that

$$
\bigcup_{n}\left\{M \in M(\mathscr{A}) \mid P_{n} \notin M\right\}
$$

is dense in the strong structure space $M(\mathscr{A})$ of $\mathscr{A}$ by the remarks at the beginning of $\S 2$. Then $C(M)=n^{-1} \cdot 1(M)+n^{-2} E_{n}(M)$ for every $M$ with $P_{n} \notin M$ and clearly 
$C(M)$ is not a scalar (zero included) multiple of the identity. But $S^{-1} C S(M)$ is not a scalar multiple of the identity for every $M$ with $P_{n} \notin M$. If for example $0 \in \mathscr{K}_{S^{-1} C S}$, then by the proof of Theorem 4 we would be able to find an invertible $T$ and a projection $E$ equivalent to 1 with $E T^{-1} C T E=0$. Thus

$$
\left\|n^{-1} E P_{n}\right\|=n^{-2}\left\|E T^{-1} E_{n} T E\right\| \leqq n^{-2}\left\|T^{-1}\right\|\|T\|
$$

for each $n=1,2, \ldots$. This is obviously impossible.

It might be well to remark that there is no canonical matrix form with 0 on the diagonal in the sense of Brown and Pearcy [2] for operators of class $\left(F^{\prime}\right)$.

LemMA 6. Let $C$ be an element in a von Neumann algebra $\mathscr{A}$. Let $D_{1}$ and $D_{2}$ be elements in $\mathscr{K}_{C}$ and let $A$ be a central element of $\mathscr{A}$ with $0 \leqq A \leqq 1$. Then $A D_{1}+$ $(1-A) D_{2} \in \mathscr{K}_{C}$.

Proof. First let $A$ be a projection in the center of $\mathscr{A}$. There are unitary operators $U_{1}, U_{2}, \ldots, U_{n}$ (respectively $V_{1}, V_{2}, \ldots, V_{m}$ ) and positive scalars $\alpha_{1}, \alpha_{2}, \ldots, \alpha_{n}$ (respectively $\beta_{1}, \beta_{2}, \ldots, \beta_{m}$ ) of sum 1 such that

$$
\left\|\sum \alpha_{i} U_{i}^{*} C U_{i}-D_{1}\right\|<\varepsilon \text { (respectively, }\left\|\sum \beta_{i} V_{i}^{*} C V_{i}-D_{2}\right\|<\varepsilon \text { ). }
$$

Here $\varepsilon>0$ is a preassigned constant. Then $U_{i}^{\prime}=U_{i} A+(1-A)(1 \leqq i \leqq n)$ and $V_{i}^{\prime}=V_{i}(1-A)+A(1 \leqq i \leqq m)$ are unitary in $\mathscr{A}$ with the property

$$
\left\|\sum \alpha_{i} \beta_{j} V_{j}^{\prime *} U_{i}^{\prime *} C U_{i}^{\prime} V_{j}^{\prime}-\left(D_{1} A+D_{2}(1-A)\right)\right\|<\varepsilon .
$$

Since $\mathscr{K}_{C}$ is closed, we have that $A D_{1}+(1-A) D_{2} \in \mathscr{K}_{C}$.

Suppose that the restriction that $A$ is a projection is removed. Let $Z$ be the spectrum of the center of $\mathscr{A}$ and let $D^{\wedge}(\zeta)$ denote the Gelfand transform of the central element $D$ evaluated at $\zeta \in Z$. Since $\mathscr{K}_{C}$ is convex, the set

is convex and so

$$
\mathscr{K}_{\zeta}=\left\{D^{\wedge}(\zeta) \mid D \in \mathscr{K}_{C}\right\}
$$

$$
A^{\wedge}(\zeta) D_{1}^{\wedge}(\zeta)+(1-A)^{\wedge}(\zeta) D_{2}(\zeta) \in \mathscr{K}_{\zeta}
$$

for every $\zeta \in Z$. Thus there is for each $\varepsilon>0$ a finite set $P_{1}, P_{2}, \ldots, P_{n}$ of orthogonal central projections of sum 1 and corresponding elements $B_{1}, B_{2}, \ldots, B_{n}$ in $\mathscr{K}_{C}$ such that

$$
\left\|\left(A D_{1}+(1-A) D_{2}-B_{j}\right) P_{j}\right\|<\varepsilon
$$

for $j=1,2, \ldots, n$. Since $\sum B_{j} P_{j} \in \mathscr{K}_{C}$ by the first paragraph and since $\varepsilon>0$ is arbitrary, we see that $A D_{1}+(1-A) D_{2}$ is in the closed set $\mathscr{K}_{C}$. Q.E.D.

The next proposition corresponds to a result of C. R. Putnam [14]. We use many of his calculations cf. $[15,1.5 .1]$.

Proposition 7. Let $A, B, C$ be elements in a properly infinite von Neumann algebra $\mathscr{A}$. If $A$ is seminormal (i.e. if either $\pm\left(A A^{*}-A^{*} A\right)$ is positive) and if $C=A B$ $-B A$, then $0 \in \mathscr{K}_{S^{-1} C S}$ for some invertible $S$ in $\mathscr{A}$. 
Proof. There is a projection $P$ in the center of $\mathscr{A}$ such that $C P$ is in the strong radical of $\mathscr{A} P$ and

$$
\{M \in M(\mathscr{A}) \mid C(1-P)(M) \neq 0\}
$$

is dense in the subset $\{M \in M(\mathscr{A}) \mid 1-P \notin M\}$ of the strong structure space $M(\mathscr{A})$ of $\mathscr{A}$. Since $\mathscr{K}_{C}=\mathscr{K}_{C P}+\mathscr{K}_{C(1-P)}=\mathscr{K}_{C(1-P)}$ due to the fact that $\mathscr{K}_{C P}=\{0\}$ [10, Proposition 2.4] and since $\{M \in M(\mathscr{A}) \mid 1-P \notin M\}$ is identified with the strong structure space of $\mathscr{A}(1-P)$, we may assume that $\{M \in M(\mathscr{A}) \mid C(M) \neq 0\}$ is dense in $M(\mathscr{A})$.

As we have previously argued we may assume that either $\mathscr{A}$ has no $\sigma$-finite type III direct summands or that $\mathscr{A}$ is the product of $\sigma$-finite type III algebras.

Now for each nonzero real scalar $\alpha$ and each unitary element $U$ in the center $\mathscr{Z}$ of $\mathscr{A}$ let

$$
F(\alpha, U)=|\alpha|^{1 / 2} U A+|\alpha|^{-1 / 2} B^{*}
$$

Then

$$
\operatorname{sgn}(\alpha)\left(F F^{*}-F^{*} F\right)=D(\alpha)+\operatorname{sgn}(\alpha) S(U)
$$

where

$$
D(\alpha)=\alpha\left(A A^{*}-A^{*} A\right)-\alpha^{-1}\left(B B^{*}-B^{*} B\right)
$$

and $S(U)=U C+U^{*} C^{*}$. The set $\left\{|D|=\left(D^{*} D\right)^{1 / 2} \mid D \in \mathscr{K}_{C}\right\}$ is monotonely decreasing in $Z$. Indeed, let $D_{1}$ and $D_{2}$ be elements of $\mathscr{K}_{C}$; there is a central projection $P$ such that $\left|D_{1}\right| P \leqq\left|D_{2}\right| P$ and $\left|D_{2}\right|(1-P) \leqq\left|D_{1}\right|(1-P)$. But $D_{1} P+D_{2}(1-P) \in \mathscr{K}_{C}$ and $\left|D_{1} P+D_{2}(1-P)\right|=\left|D_{1}\right| P+\left|D_{2}\right|(1-P)$ is majorized by both $\left|D_{1}\right|$ and $\left|D_{2}\right|$. This proves that $\left\{|D| \mid D \in \mathscr{K}_{C}\right\}$ is monotonely decreasing. Let $D_{0}$ be the greatest lower bound of this set [6, Appendix II]. Suppose $D_{1}$ is a positive central element which majorizes $D(\alpha)$ for some $\alpha$. We show that $D_{0} \leqq D_{1}$. If not, there is an $\varepsilon>0$ and a nonzero central projection $P$ such that $D_{0} P \geqq\left(D_{1}+\varepsilon\right) P$. By reducing to $\mathscr{A} P$ we may assume that $P=1$. Let $U_{1}, U_{2}, \ldots, U_{n}$ be unitary elements in $\mathscr{Z}$ and let $\alpha_{1}, \alpha_{2}, \ldots, \alpha_{n}$ be positive scalars of sum 1 ; then for any central element $R$ in the sphere of radius $2^{-1} \varepsilon$ about 0 we have that

$$
\left|\left(\sum \alpha_{i} U_{i}\right) D_{1}+R\right|^{2} \leqq\left(\sum \alpha_{i} D_{1}+|R|\right)^{2}=\left(D_{1}+|R|\right)^{2} \leqq\left(D_{1}+2^{-1} \varepsilon\right)^{2} .
$$

Hence $\left(D^{*} D x, x\right) \leqq\left(\left(D_{1}+2^{-1} \varepsilon\right)^{2} x, x\right)$ for any $D$ in the strong closure $\mathscr{K}^{\prime \prime}$ of the convex hull of the set

$$
\left\{U D_{1}+R \mid U, R \text { in } \mathscr{Z}, U \text { unitary, }\|R\| \leqq 2^{-1} \varepsilon\right\}
$$

and for any $x$ in the Hilbert space $H$ of $\mathscr{A}$. On the other hand we see that $\left(D^{*} D x, x\right)$ $\geqq\left(\left(D_{1}+\varepsilon\right)^{2} x, x\right)$ for any $D$ in the strong closure $\mathscr{K}^{\prime}$ of $\mathscr{K}_{C}$ and any $x$ in $H$ because $R^{*} R \geqq\left(D_{1}+\varepsilon\right)^{2}$ for every $R$ in $\mathscr{K}_{C}$. By the standard separation theorem there is a nonzero strongly continuous functional $f$ on $\mathscr{Z}$ such that

$$
\operatorname{lub}\left\{\operatorname{Re} f(R) \mid R \in \mathscr{K}^{\prime}\right\} \leqq \operatorname{glb}\left\{\operatorname{Re} f(R) \mid R \in \mathscr{K}^{\prime \prime}\right\} .
$$


Here $\operatorname{Re} \beta$ denotes the real part of the complex number $\beta$. Indeed the element 0 is not in the strong closure of $\mathscr{K}^{\prime}-\mathscr{K}^{\prime \prime}$. Since $f$ is also weakly continuous on $\mathscr{Z}$ [6, I, $\S 3$, Theorem 1 (i)], there is a unitary $U$ in $\mathscr{Z}$ and a nonzero vector $x$ in $H$ such that $f(R)=(R U x, x)$ for every $R \in \mathscr{Z}[17]$ and [6, III, $\$ 1$, Corollary, Theorem 4]. Now let $F=F(\alpha, \operatorname{sgn}(\alpha) U)$. We have that

$$
\operatorname{sgn}(\alpha)\left(F F^{*}-F^{*} F\right)=D(\alpha)+S(U) \leqq D_{1}+S(U) .
$$

Let $D$ be an arbitrary element in $\mathscr{K}_{\operatorname{sgn}(\alpha)\left(F F^{*}-F^{*} F\right)}=\mathscr{K}$. There is an element $S$ in $\mathscr{K}_{S(U)}$ such that $D \leqq D_{1}+S\left[6, \mathrm{III}, \S 5\right.$, Problem 2a]. We may find a $T$ in $\mathscr{K}_{U C-U^{*} C^{*}}$ such that

$$
2^{-1}(S+T) \in \mathscr{K}_{U C} \quad \text { and } \quad 2^{-1}(S-T) \in \mathscr{K}_{U \cdot C^{*}}
$$

$\left[6\right.$, III, $\S 5$, Problem 2a]. We then have that $2^{-1} U^{*}(S+T)$ and $2^{-1} U^{*}(S-T)^{*}$ are elements of $\mathscr{K}_{C}$. The latter is true because $\mathscr{K}_{C^{*}}=\left\{R^{*} \mid R \in \mathscr{K}_{C}\right\}$. From relation (7) we obtain that both $\operatorname{Re}\left(2^{-1}(S+T) x, x\right)$ and $\operatorname{Re}\left(2^{-1}(S-T) x, x\right)$ are majorized by glb $\left\{\operatorname{Re} f(R) \mid R \in \mathscr{K}^{\prime \prime}\right\}$. Thus

$$
(S x, x)=\operatorname{Re}(S x, x) \leqq 2 \operatorname{glb}\left\{\operatorname{Re} f(R) \mid R \in \mathscr{K}^{\prime \prime}\right\}
$$

since $S$ is clearly selfadjoint. But $-U^{*} D_{1}-2^{-1} \varepsilon U^{*}$ is an element of $\mathscr{K}^{\prime \prime}$. So

$$
2^{-1}(S x, x) \leqq \operatorname{Re}\left(U\left(-U^{*} D_{1}-2^{-1} \varepsilon U^{*}\right) x, x\right)=-\left(\left(D_{1}+2^{-1} \varepsilon\right) x, x\right) .
$$

Therefore,

$$
(D x, x) \leqq\left(D_{1} x, x\right)-\varepsilon(x, x)-2\left(D_{1} x, x\right) \leqq-\varepsilon(x, x)
$$

by relation (8). Using reasoning similar to that which we used to prove that $\left\{|R| \mid R \in \mathscr{K}_{C}\right\}$ is monotonely decreasing, we may prove that $\mathscr{K}$ is monotonely increasing. Setting $R_{0}=$ lub $\mathscr{K}$, we see that $\left(R_{0} x, x\right) \leqq-\varepsilon(x, x)$. We show that this is impossible by showing $\left(R_{0} x, x\right) \geqq 0$. Indeed, in proving this then we may certainly assume that $F$ is invertible and that $\alpha>0$. Because $F$ is invertible, there is a unitary operator $V$ in $\mathscr{A}$ obtained from the polar decomposition of $F$ [6, Appendix III] such that $V^{*} F F^{*} V=F^{*} F$. If $R \in \mathscr{K}_{F^{*} F}$, then there are unitary operators $U_{1}, U_{2}, \ldots, U_{n}$ in $\mathscr{A}$ and positive scalars $\alpha_{1}, \alpha_{2}, \ldots, \alpha_{n}$ of sum 1 such that

$$
\left\|\sum \alpha_{i} U_{i}^{*} F^{*} F U_{i}-R\right\|<\varepsilon^{\prime}
$$

for any preassigned constant $\varepsilon^{\prime}>0$. But this means that

$$
\left\|\sum \alpha_{i}\left(V U_{i}\right)^{*} F F^{*}\left(V U_{i}\right)-R\right\|<\varepsilon^{\prime} .
$$

Because $\varepsilon^{\prime}>0$ is arbitrary we have that $R \in \mathscr{K}_{F F^{*}}$. By symmetry it is then clear that $\mathscr{K}_{F^{*}}=\mathscr{K}_{F^{*} F}$. Now for any $\varepsilon^{\prime}>0$ there is an element $R_{1}$ in $\mathscr{K}_{F F^{*}}$ such that

$$
\left(R_{1} x, x\right) \geqq\left(R_{2} x, x\right)-\varepsilon^{\prime}(x, x)
$$

where $R_{2}$ is the least upper bound of the monotonely increasing set $\mathscr{K}_{F F^{*}}$. But 
there is an element $R \in \mathscr{K}_{F^{*} F}$ such that $R_{1}-R \in \mathscr{K}[6,111, \S 5$, Problem 2a]. However we have that

$$
\left(R_{0} x, x\right) \geqq\left(\left(R_{1}-R\right) x, x\right) \geqq\left(R_{2} x, x\right)-(R x, x)-\varepsilon^{\prime}(x, x) \geqq-\varepsilon^{\prime}(x, x)
$$

since $R_{2} \geqq R$. Because $\varepsilon^{\prime}>0$ is arbitrary, we see that $\left(R_{0} x, x\right) \geqq 0$. This is a contradiction. We must conclude that $D_{0} \leqq D_{1}$.

We now show that $D_{0}=0$. We may assume that $A A^{*}-A^{*} A \leqq 0$. Then for $\alpha>0$ we have that

$$
D(\alpha) \leqq-\alpha^{-1}\left(B B^{*}-B^{*} B\right) \leqq 2 \alpha^{-1}\|B\|^{2} .
$$

Thus we see that $D_{0} \leqq 2 \alpha^{-1}\|B\|^{2}$ for every $\alpha>0$. Therefore $D_{0}=0$. If $Q$ is a nonzero central projection in $\mathscr{A}$ and if $\varepsilon>0$ there is a net $\left\{Q_{n}\right\}$ of mutually orthogonal central projections of sum $Q$ such that each set $\mathscr{K}_{C Q_{n}}$ contains an element $D_{n}$ of norm not exceeding $\varepsilon$. Indeed, if $\left\{Q_{n}\right\}$ is a maximal set of mutually orthogonal nonzero central projections majorized by $Q$ with this property, then the assumption that $Q^{\prime}=Q-\sum Q_{n} \neq 0$ gives a contradiction. Since glb $\left\{|D| \mid D \in \mathscr{K}_{C Q^{\prime}}\right\}=0$, there is a $D \in \mathscr{K}_{C Q^{\prime}}$ such that $|D| \geqq 2^{-1} \varepsilon Q^{\prime}$ is not true. This means that there is a nonzero central projection $Q^{\prime \prime}$ majorized by $Q^{\prime}$ such that $|D| Q^{\prime \prime} \leqq \varepsilon Q^{\prime \prime}$. This contradicts the maximality of $\left\{Q_{n}\right\}$. Hence we have that $\sum Q_{n}=Q$.

Now suppose $\mathscr{A}$ has no $\sigma$-finite type III direct summands. In Theorem 4 we constructed a sequence $\left\{P_{n}\right\}$ of mutually orthogonal central projections of sum 1 and a projection $F$ whose orthogonal complement $1-F$ was in the strong radical such that $\|(C-R) F P\| \leqq 260 v(C P)$ whenever $P$ is a central projection majorized by $P_{n}$ and whenever $R \in \mathscr{K}_{C P}$. Also either $P_{n}=0$ or $\nu(C P) \in\left[2^{-n}\|C\|, 2^{-n+1}\|C\|\right]$ for every nonzero central projection $P$ majorized by $P_{n}$. By the preceding paragraph there is a set $\left\{P_{n j}\right\}$ of mutually orthogonal central projections of sum $P_{n}$ such that each set $\mathscr{K}_{C P_{n j}}$ contains an element $D_{n j}$ of norm not exceeding $\nu\left(C P_{n}\right)$. Then for each nonzero central projection $P$ majorized by $P_{n}$ we have that

$$
\begin{aligned}
\|C F P\| & =\operatorname{lub}_{j}\left\|C F P P_{n j}\right\| \leqq \operatorname{lub}\left\|\left(C-D_{n j}\right) P P_{n j}\right\|+\operatorname{lub}\left\|D_{n j} P P_{n j}\right\| \\
& \leqq 260 \nu(C P)+\nu\left(C P_{n}\right) \leqq 262 \nu(C P)
\end{aligned}
$$

by relation (6). By the same reasoning as found in Theorem 4, we may find an invertible $W$ in $\mathscr{A}$ such that $E W^{-1} C W E=0$ for some projection $E$ in $\mathscr{A}$ which is equivalent to 1 . However, this means that $0 \in \mathscr{K}_{\mathrm{W}^{-1} \mathrm{CW}}$ by Proposition 5 .

If $A$ is the product of $\sigma$-finite type III algebras a similar proof holds. Q.E.D.

COROllaRY. If $F$ is an element in a properly infinite von Neumann algebra $\mathscr{A}$, then there is an invertible $S$ in $\mathscr{A}$ such that $\mathscr{K}_{S^{-1}\left(F^{*} F-F F^{*}\right) S}$ contains 0.

Proof. If $A=2^{-1} i\left(F-F^{*}\right)$ and $B=2^{-1}\left(F+F^{*}\right)$, then $2^{-1} i\left(F F^{*}-F F^{*}\right)=A B$ $-B A$. Now Proposition 7 applies.

Added in proof (April 25, 1970). I have improved Proposition 7 by showing that $0 \in \mathscr{K}_{C}$. 


\section{BIBLIOGRAPHY}

1. A. Brown, P. Halmos and C. Pearcy, Commutators of operators on Hilbert space, Canad. J. Math. 17 (1965), 695-708. MR 34 \#3311.

2. A. Brown and C. Pearcy, Structure of commutators of operators, Ann. of Math. (2) 82 (1965), 112-127. MR 31 \#2612.

3. - Commutators in factors of type III, Canad. J. Math. 18 (1966), 1152-1160. MR 34 \#1864.

4. A. Brown, C. Pearcy and D. Topping, Commutators and the strong radical, Duke Math. J. 35 (1968), 853-859. MR 38 \#1534.

5. D. Deckard and C. Pearcy, On continuous matrix-ralued functions on a Stonian space, Pacific J. Math. 14 (1964), 857-869. MR 30 \#2356.

6. J. Dixmier, Les algèbres d'opérateurs dans l'espace hilbertien, Cahiers Scientifiques, fasc. 25, Gauthier-Villars, Paris, 1957. MR 20 \#1234.

7. - Les $C^{*}$-algèbres et leurs représentations, Cahiers Scientifiques, fasc. 29, GauthierVillars, Paris, 1964. MR 30 \#1404.

8. J. M. G. Fell, The structure of algebras of operator fields, Acta Math. 106 (1961), 233-280. MR 29 \#1547.

9. J. Glimm, A Stone-Weierstrass theorem for $C^{*}$-algebras, Ann. of Math. (2) 72 (1960), 216-244. MR $22 \# 7005$.

10. H. Halpern, Commutators in properly infinite von Neumann algebras, Trans. Amer. Math. Soc. 139 (1969), 55-73.

11. - Irreducible homomorphisms of a von Neumann algebra into its center, Trans. Amer. Math. Soc. 140 (1969), 195-221. MR 39 \#3322.

12. G. Lumer and M. Rosenblum, Linear operator equations, Proc. Amer. Math. Soc. 10 (1959), 32-41. MR 21 \#2927.

13. Y. Misonou, On a weakly central operator algebra, Tôhoku Math. J. (2) 4 (1952), 194-202. MR 14, 566.

14. C. R. Putnam, On commutators of bounded matrices, Amer. J. Math. 73 (1951), 127-131. MR 12, 836.

15. - Commutation properties of Hilbert space operators and related topics, Ergebnisse der Mathematik und ihrer Grenzgebiete, Band 36, Springer-Verlag, New York, 1967. MR 36 \#707.

16. C. Rickart, General theory of Banach algebras, The University Series in Higher Math., Van Nostrand, Princeton, N. J., 1960. MR 22 \#5903.

17. S. Sakai, On linear functionals of $W^{*}$-algebras, Proc. Japan Acad. 34 (1958), 571-574. MR 21 \#5915.

IlLinOIS InSTITUte OF TeChNOLOGY, Chicago, Illinois 60616 